\title{
Simple Model for Overcharging of a Sphere by a Wrapped Oppositely Charged Asymmetrically Neutralized Polyelectrolyte: Possible Effects of Helical Charge Distribution
}

\author{
Andrey G. Cherstvy* and Roland G. Winkler \\ Institut für Festkörperforschung, Forschungszentrum Jülich, D-52425 Jülich, Germany \\ Received: August 20, 2004; In Final Form: November 26, 2004
}

\begin{abstract}
We investigate the complexation of a polyelectrolyte bendable rod with an oppositely charged spherical macroion. We take into account electrostatic bending of the rod and its asymmetric charge neutralization by sphere charges. The spontaneous curvature of the rod toward the sphere results in a substantial overcharging of such polyelectrolyte complex with a possible phase transition. Assuming a discrete helical charge distribution on the rod surface, we calculate the electrostatic energy of the helix and the electrostatic contribution to its bending and twisting elasticity. We show that the latter may change sign when the helical pitch is changed. For a DNA-relevant case, these corrections appear to be small compared to the corresponding mechanical elastic moduli. We discuss possible applications of our results to the description of overcharging of the nucleosome core particles.
\end{abstract}

\section{Introduction}

Nucleosome core particles (NCPs), the elementary units of the chromatin, play a fundamental role in many biological processes. ${ }^{1}$ Eukaryotic genomic DNA is (evolutionary) constructed to help DNA packaging and to govern the positioning of NCPs along the DNA. ${ }^{2}$ It is known that particular DNA sequences have a higher affinity to the histone core, whereas long stretches of poly $(\mathrm{dA}) \cdot \operatorname{poly}(\mathrm{dT})$, for instance, prevent the nucleosome formation. ${ }^{3}$ The structure of the NCP is highly conserved in different organisms and tissues. ${ }^{4}$

DNA adapts its structure to fit the highly bent state in the $\mathrm{NCP}$, where the DNA radius of curvature is only twice as large as the DNA diameter. ${ }^{5-7}$ In particular, DNA is overtwisted in the NCP by $0.3-0.5 \mathrm{bp} / \mathrm{turn}$, on average, ${ }^{5,8-10}$ compared to DNA in solution with $10.5 \mathrm{bp} / \mathrm{turn} .{ }^{11}$ DNAs use their sequencedependent bendability and twistability ${ }^{12}$ to achieve a better packaging in the NCP. (The CG (AT) have a preference to bend into the major (minor) DNA groove. ${ }^{2,8}$ ) Also, trinucleotides AAA and TTT are found more often when the minor groove faces the octamer, whereas GGC and GCC are in the minor groove when it points outward the NCP. ${ }^{13}$ Several models, mostly stereochemical, of such "DNA kinking" have been suggested in the literature. ${ }^{14-17}$ Although the structure of NCP has been resolved recently with a great accuracy by X-ray diffraction on NCP crystals, ${ }^{6,8,18}$ the physical basics and the factors governing NCP stability in solution are not yet completely understood.

Electrostatics. The DNA wrapped in the NCP is known to overcharge considerably the histone protein core. As both the histone core (rich in lysine and arginine) and the DNA are strongly and oppositely charged objects, the electrostatic interaction should play an important role in their complexation (although specific DNA-histone interactions should not be overlooked). The influence of salt conditions on the NCP stability supports this hypothesis: DNA is released from the NCP for salt concentrations outside the physiological range. ${ }^{19,20}$ The NCP structure also suggests that close contacts of DNA with the histone proteins may result in neutralization on the

* Corresponding author. E-mail: a.cherstvy@fz-juelich.de. inner-to-core DNA phosphates. Possible consequences of such neutralization have been predicted long $\mathrm{ago}^{21}$ and explored within a simple electrostatic model of rod bending. ${ }^{22}$ However, DNA interactions with the core and the DNA helical symmetry have not been included in these models. (DNA helicity was however shown to be important in all-atom computer simulations of the bending of asymmetrically neutralized DNA. ${ }^{23}$ )

Various theoretical electrostatic models of complexation of polyelectrolyte chains of different flexibilities with oppositely charged sphere(s) have been suggested to model DNA-histone complexes. ${ }^{24-33,35}$ Transitions between wrapped and unwrapped conformations for relatively short chains ${ }^{26}$ as well as transitions between touching, bent, and completely wrapped conformations for long chains ${ }^{29}$ have been predicted. Rosette-like chain-sphere complexes for the chain with high bending rigidity have also been described. ${ }^{25,34}$ The complex formation has been studied by computer simulations ${ }^{36-39}$ and experimentally; ${ }^{40}$ however, some of its features still remain unclear. In particular, how strong can be the curvature induced by an asymmetric neutralization of charges of the wrapped rod, and how can this affect the charge of the complex? How does the DNA helicity contribute to this effect?

In this work, we extend the treatment of ref 22 to include the electrostatic interaction of an asymmetrically neutralized rod with an oppositely charged sphere. We show the quantitative effects of spontaneous rod curvature and calculate the corresponding degree of sphere overcharging in section II. Assuming a helical distribution of discrete charges on the rod, we estimate the electrostatic contribution to its twisting and bending elasticity in section III. We sum the Debye-Hückel interaction potentials to calculate the electrostatic interaction energy of the charges, as it was suggested in refs 41 and 42 . Finally, we compare our results with the results of existing theoretical models and discuss the differences between them in section IV.

\section{Spontaneous Rod Curvature and Sphere Overcharging}

Model and Approximations. We adapt a highly simplified model of a DNA-sphere complex. The complex consists of a bendable polyelectrolyte rod of radius $a$ wrapped around an 

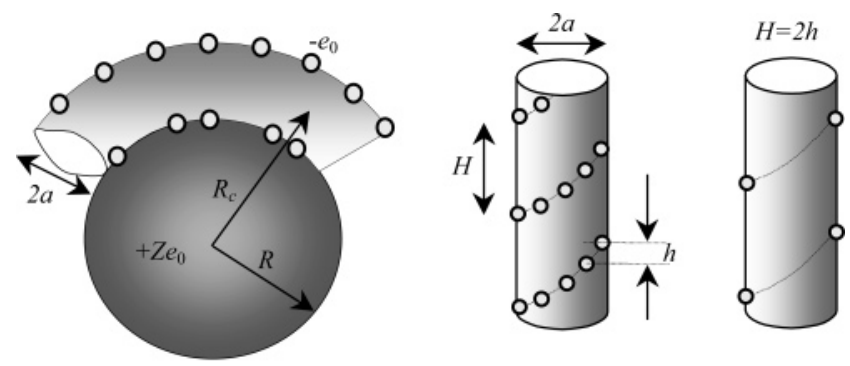

a

b

Figure 1. Structure of the complex of a rod wrapped around an oppositely charged sphere (a) and the parameters of the helix with discrete charges (b, c). The nucleosome is a cylinder of about $110 \AA$ in diameter and $60 \AA$ in height.

oppositely charged sphere of radius $R$ and charge $Z e_{0}$, as shown in Figure 1a. The straight rod carries two identical linear arrays of elementary charges $\left(-e_{0}\right)$, with the charge-charge separation $h$, which are located on opposite sides of the rod. When the rod is bent around the (incompressible) sphere, one array of charges is in contact with the sphere. Thus, the charge density increases on the inner-to-sphere rod surfaces and decreases on the outer-to-sphere one. If some of the inner-to-sphere rod charges are neutralized by sphere charges, the repulsion between them is diminished. The repulsion of the outer-to-sphere charges induces a rod curvature toward the sphere that favors rod adsorption and may result in overcharging of the sphere (a curved macroion).

We approximate the interactions among the charges by the Debye-Hückel potential, which captures the actual screened charge-charge interactions. ${ }^{24}$ We assume that the fraction (1 $-\eta$ ) of the macroion charges are mobile and that they can participate in neutralization of inner-to-sphere rod charges. Rod-sphere electrostatic interactions are thus effectively renormalized upon the rod adsorption. We consider the limit of a persistent rod (rod persistence length $l_{p} \gtrsim R$ ), neglecting rod fluctuations and other entropic contributions. ${ }^{43} \mathrm{We}$ first consider the energy of an asymmetrically neutralized rod alone and then address the rod-sphere interaction.

A. Asymmetrically Neutralized Rod. a. Straight Rod. The electrostatic (el) energy $E_{\mathrm{el}}^{\text {line }}$ per length $h$ of an infinite straight array of pointlike charges interacting via the Debye-Hückel potential is 27,44

$$
\frac{E_{\mathrm{el}}^{\mathrm{line}}}{k_{\mathrm{B}} T l_{\mathrm{B}}}=\sum_{n=1}^{\infty} \frac{\mathrm{e}^{-\kappa n h}}{n h}=-\frac{\ln \left[1-\mathrm{e}^{-\kappa h}\right]}{h}
$$

Here $l_{\mathrm{B}}=e_{0}^{2} /\left(\epsilon k_{\mathrm{B}} T\right)$ is the Bjerrum length $\left(k_{\mathrm{B}}\right.$ is the Boltzmann constant, $T$ is the absolute temperature, $\epsilon$ is the dielectric constant of water), and $\kappa$ is the reciprocal Debye screening length of the solution. We use this kind of summation for different charge configurations several times in this paper.

In a free (f) state, the energy of a straight rod of the length $L$ with two linear arrays of charges is $E_{\mathrm{el}}^{\text {rod,f }} /\left(k_{\mathrm{B}} T L l_{\mathrm{B}}\right)=-2 \ln [1$ $\left.-\mathrm{e}^{-\kappa h}\right] / h^{2}$. The interaction energy between these two arrays is neglected below for simplicity because it is nearly the same for a straight and a bent rod conformation. The electrostatic energy of a bent (b) line, $E_{\mathrm{el}}^{\text {line,b }}$, with the curvature radius $R_{\mathrm{c}}$ is

$$
\frac{E_{\mathrm{el}}^{\mathrm{line}, \mathrm{b}}}{k_{\mathrm{B}} T L l_{\mathrm{B}}}=\sum_{n=1}^{\infty} \frac{\mathrm{e}^{-\kappa \mathrm{r}_{n}}}{h r_{n}}, \quad r_{n}=2 R_{\mathrm{c}} \sin \left[\frac{h n}{2 R_{\mathrm{c}}}\right]
$$

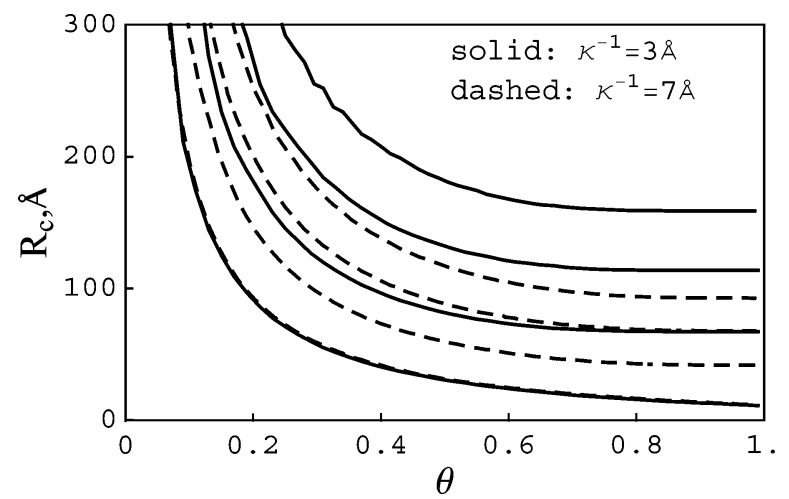

Figure 2. Radius of spontaneous curvature of an asymmetrically neutralized rod. Parameters: $a=10 \AA, h=3.4 \AA, \kappa^{-1}=3 \AA$ (solid) and $\kappa^{-1}=7 \AA$ (dashed), $l_{\mathrm{p}}=750 \AA, 500 \AA, 250 \AA$, and 0 (from top to bottom).

where $r_{n}$ is the distance between two charges separated by $n h$ along the rod axis.

b. Bent Rod. We presume that some of the rod charges located next to the sphere are neutralized by the mobile sphere charges. The remaining rod charges are allowed to redistribute: the mean charge-charge separation increases by $1 /(1-$ $\theta$ ), where $\theta$ is the fraction of neutralized inner rod charges (see Figure 1). For $\kappa h \ll 1$ (the screening length comprises many rod charges) and for large curvature radii, ${ }^{24,41}$ the energy of the bent $\operatorname{rod} E_{\mathrm{el}}^{\text {rod,b }}(2)$ can be approximated as ${ }^{43,45}$

$$
\begin{aligned}
& \frac{E_{\mathrm{el}}^{\mathrm{rod}, \mathrm{b}}}{k_{\mathrm{B}} T L l_{\mathrm{B}}} \approx-\frac{\ln \left[1-\mathrm{e}^{-\kappa h_{+}}\right]}{h h_{+}}-\frac{\ln \left[1-\mathrm{e}^{-\kappa h /(1-\theta)}\right]}{h h_{-} /(1-\theta)^{2}}+ \\
& \frac{1}{4 \kappa^{2} h^{2}}\left(\frac{h}{2\left(R_{\mathrm{c}}+a\right)^{2} h_{+}}+\frac{h(1-\theta)^{2}}{2\left(R_{\mathrm{c}}-a\right)^{2} h_{-}}\right)
\end{aligned}
$$

Here, $h_{ \pm}=h\left(R_{\mathrm{c}} \pm a\right) / R_{\mathrm{c}}$ are the charge-charge separations on inner $(-)$ and outer $(+)$ to sphere rod charge arrays. Below, we will use this expression as an approximation, although the actual rod curvature radii might be comparably small. Note that the last term in eq 3 is similar to the Odijk-Skolnick-Fixman correction for the persistence length of a rodlike polyelectrolyte chain in salt solution ${ }^{46}$

$$
l_{\mathrm{el}}^{\text {line }}=l_{\mathrm{B}} /\left(4 \kappa^{2} h^{2}\right)
$$

The mechanical bending energy of the $\operatorname{rod}$ is $E_{\mathrm{bend}}^{\mathrm{rod}, \mathrm{b}} /\left(k_{\mathrm{B}} T L\right)=$ $l_{\mathrm{p}} /\left(2 R_{\mathrm{c}}{ }^{2}\right)$. We use the model of a homogeneous rod bending, although for small $R_{\mathrm{c}}$ some modifications of the bending mechanism are possible (rod kinking, ${ }^{47}$ etc.).

The results of minimization with respect to the radius of curvature $R_{\mathrm{c}}$ of the total energy difference between the bent and free state are shown in Figure 2 for a rod with parameters close to those of $B$-DNA. The value of the spontaneous curvature radius of the rod, $R_{\mathrm{c}}$, decreases with increasing $\theta$ since less contraction of inner-to-sphere charges is needed upon bending. At larger salt content (larger $\kappa$ ) $R_{\mathrm{c}}$ increases because the electrostatic interaction becomes weaker. The value of $R_{\mathrm{c}}$ decreases with decreasing $l_{\mathrm{p}}$, as one could expect (see Figure 2 ). The energy depth (the difference of the total energy for the state with $R_{\mathrm{c}}=\infty$ and with the optimal $R_{\mathrm{c}}$ ) varies for $\theta=0.5$ from $\approx 0.02 k_{\mathrm{B}} T / \AA$ for $l_{\mathrm{p}}=500 \AA$ at $\kappa^{-1}=3 \AA$ to $\approx 0.15 k_{\mathrm{B}} T / \AA$ for $l_{\mathrm{p}}=0$ at $\kappa^{-1}=7 \AA$. If $R=R_{\mathrm{c}}$, no bending energy is required to wrap a rod around the sphere. If $R>R_{\mathrm{c}}$, the rod is wrapped spontaneously; i.e., it has a negative persistence length, and the adsorption can continue beyond the isoelectric point of the complex. 
Note that a partial neutralization of the rod charges (by condensed/adsorbed cations) would diminish the value of the electrostatic bending persistence length. In particular, for a sequence of alternating $-e_{0}$ and $+\alpha e_{0}$ charges, this value is $l_{\mathrm{el}, \pm}^{\text {line }} \approx l_{\mathrm{B}}(1-\alpha)^{2} /\left(4 \kappa^{2} b^{2}\right)$, where $b$ is the distance between the closest positive and negative charges. Note also that the screening length inside the cylinder core can be larger than $\kappa^{-1}$, as in DNA-histone complexes, because the core contains almost no mobile ions. Thus, the charges on the outer surface of the bent rod, interacting through the core, repel each other stronger than those on the inner surface. This can be the another source of spontaneous rod curvature that occurs without an asymmetric charge neutralization.

B. Rod-Sphere Interactions. We assume that the sphere charge is renormalized by the rod adsorption that prevents the overcharging of the complex due to pure rod-sphere interactions. We estimate how the effective charge of the complex changes due to the additional curvature of the asymmetrically neutralized rod considered in the last subsection. Let the fraction $\eta$ of sphere charges be immobile. For the bound (b) state of the rod of the length $L$ the interaction energy with the sphere (sp) can be written as

$$
\frac{E_{\mathrm{el}}^{\mathrm{sp}-\text { rod,b }}}{k_{\mathrm{B}} T L l_{\mathrm{B}}}=-\frac{(Z-2 L / h)}{h(1+\kappa R)}\left[\frac{\mathrm{e}^{-2 \kappa a}}{R+2 a}+\frac{1-\theta}{R}\right]
$$

Here $\theta$ is the fraction of compensated charges on the inner rod surface; $\theta=Z(1-\eta) h / L<1$ if all mobile sphere charges are required to neutralize the inner rod charges, and $\theta=1$ if there is an excess of mobile sphere charges after the neutralization of all inner rod charges. The terms in eq 5 are the attraction of the outer and of the partially compensated inner rod charges to the sphere, respectively. Here $L_{*}=Z(1-\eta) h$ is the length of the rod with all the inner-to-sphere charges neutralized by the mobile sphere charges. Equation 5 is constructed so that the overcharging presented in Figure 3 is only due to the additional bending of asymmetrically neutralized rod toward the sphere. In the free state, $E_{\mathrm{el}}^{\mathrm{sp}-\text { rod,f }}=0$. Note that we do not solve the Poisson-Boltzmann equation to calculate the potential of the complex and its energy here; instead, we use the approximate expressions for the interaction and self-energy of the complex.

The sphere self-energies in the bound and free state

$$
\frac{E_{\mathrm{el}}^{\mathrm{sp}, \mathrm{b}}}{k_{\mathrm{B}} T l_{\mathrm{B}}}=\frac{(Z-2 L / h)^{2}}{2 R(1+\kappa R)} \quad \text { and } \quad \frac{E_{\mathrm{el}}^{\mathrm{sp}, \mathrm{f}}}{k_{\mathrm{B}} T l_{\mathrm{B}}}=\frac{Z^{2}}{2 R(1+\kappa R)}
$$

favor neutral sphere-rod complexes.

For large sphere radii and large rod charge densities, the length of the wrapped rod can be smaller than the circumference of the sphere. In general, however, several turns of a wrapped rod may correspond to the energy minimum. Then, the repulsion between the charges of different turns can be approximated by the repulsion of straight lines, using the potential $e_{0} \psi(x) /\left(k_{\mathrm{B}} T\right)$ $\approx 2\left(l_{\mathrm{B}} / h\right) K_{0}(x)$, where $K_{0}(x)$ is the modified Bessel function. This can decrease the degree of overcharging predicted below. For simplicity, the parameters in Figure 3 are chosen such that less than one turn of the rod is usually wrapped, $L<$ $2 \pi(R+a)$. Note that in DNA-relevant cases the interaction between the turns must not necessarily be repulsive (see section IV). Note also that the full solution of the linearized PoissonBoltzmann theory for adsorption of a thin polyelectrolyte chain on the oppositely charged sphere, where the chain configurations with several turns have been considered, results in undercharged complexes. ${ }^{35}$
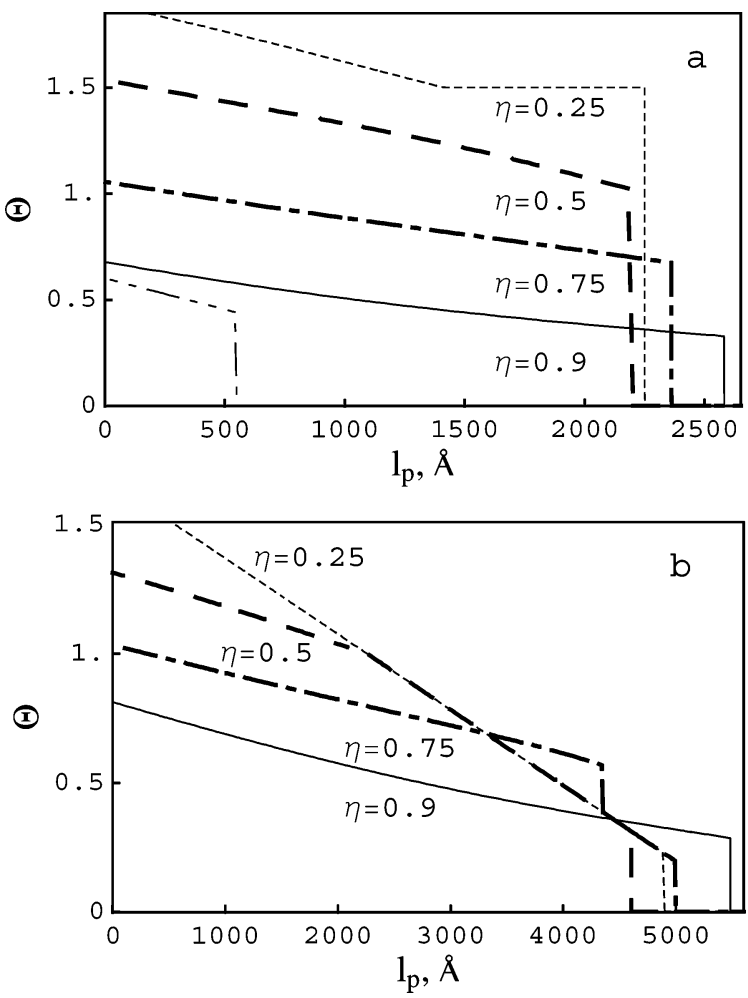

Figure 3. Fraction $\Theta$ of the sphere charges compensated by the adsorbed rod vs persistence length $l_{\mathrm{p}}$. Parameters: $a=10 \AA, \kappa^{-1}=3$ $\AA, h=3.4 \AA, R=50 \AA, Z=100$. For (b) the sphere self-energy (6) is counted, whereas for (a) it is neglected. In (a), the dot-dot-dashed curve corresponds to the simple case of rod adsorption to the sphere when no repulsion within the rod is considered (at $\eta=0.9$ ).

Charge of the Complex. The minimization of the energy difference between the bound and free state

$$
\begin{aligned}
\Delta E(L)=E_{\mathrm{el}}^{\mathrm{rod}, \mathrm{b}}-E_{\mathrm{el}}^{\mathrm{rod}, \mathrm{f}}+E_{\mathrm{el}}^{\mathrm{sp}-\mathrm{rod}, \mathrm{b}}- & E_{\mathrm{el}}^{\mathrm{sp}-\mathrm{rod}, \mathrm{f}}+ \\
& E_{\mathrm{el}}^{\mathrm{sp}, \mathrm{b}}-E_{\mathrm{el}}^{\mathrm{sp}, \mathrm{f}}+E_{\mathrm{bend}}^{\mathrm{rod}, \mathrm{b}}
\end{aligned}
$$

yields the optimal length $L$ of the rod adsorbed on the sphere and the total fraction of neutralized sphere charges (Figure 3)

$$
\Theta=2 L /(Z h)
$$

In the simplest case, a thin polyelectrolyte chain adsorbs on the oppositely charged sphere only if the gain in attractive interaction energy is larger than the bending energy penalty, i.e., when ${ }^{25} l_{\mathrm{p}} \lesssim 2 Z l_{\mathrm{B}} /(h \kappa)$. For the adsorption of the rod with two charged strings this critical adsorption condition turns into $l_{\mathrm{p}}<l_{\mathrm{p}} * \approx\left[2 Z l_{\mathrm{B}}(R+a)^{2} / h(1+\kappa R)\right]\left[\mathrm{e}^{-2 \kappa a} /(R+2 a)+(1-\right.$ $\theta) / R]$. The fraction of compensated charges of the sphere is always smaller than unity (undercharging). The neutralization of inner-to-sphere rod charges dramatically modifies this picture. For $\eta$ close to unity, there are not enough mobile charges to neutralize the inner rod charges, and the complex reveals no overcharging. However, for smaller $\eta$ the complex is substantially overcharged due to the additional rod bending. Wrapping occurs at relatively large $l_{\mathrm{p}}$ since the bare rod charge and sphere charge were chosen to be quite large, and no counterion condensation effects ${ }^{44}$ were considered. The energy $\Delta E(L)$ may have two minima, and the transition between them may occur abruptly, at some critical $l_{\mathrm{p}}$ (Figure $3 \mathrm{~b}$ ). The energy depth for an optimal $L$ is large for small $l_{\mathrm{p}}$, it decreases with increasing rod stiffness, and it vanishes near the transition points. The dependence of $\Theta$ on $\kappa$ (Figure 4) reveals that at very low ionic 


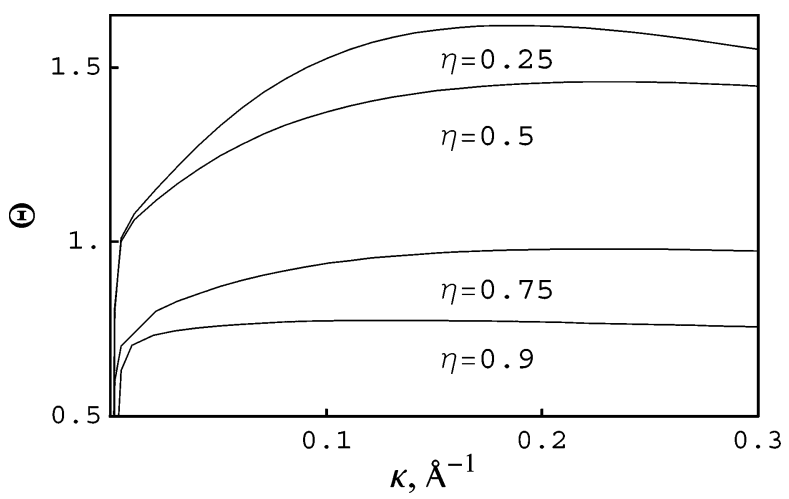

Figure 4. Fraction of the sphere charge neutralization as a function of inverse screening length of the solution. The parameters are same as in Figure 3b, except for $l_{\mathrm{p}}=500 \AA$.

strength the charge of the complex goes to zero since $l_{\mathrm{el}}^{\text {line }}$ rapidly grows, and the adsorption becomes unprofitable. For large ionic strengths, the charge of the complex also decreases since the electrostatic interactions, bending the rod and attracting it to the sphere, become weaker.

Note that one can do similar estimates for several charged lines on the rod surface, as a better model for the DNA charge pattern, and also include the sphere compressibility. In this case, we also expect to observe an overcharging although possibly diminished. It is also possible that some particular conformations of the helical charge array on the surface of the macroion might be favored. This subject requires a more delicate consideration of the energy balance, including the helix-helix interaction, which is beyond the scope of the present paper.

\section{Bending and Twisting a Helix of Discrete Charges}

The results of the previous section can be relevant for NCP overcharging; however, the helical symmetry of DNA may affect them to some extent. First, the bending elasticity of the helix is not the same as that of the linear array of the same charge density. Second, DNA helicity introduces another type of deformation, namely twisting. In this section, we estimate the electrostatic bending $l_{\mathrm{el}}^{\mathrm{hel}}$ and twisting $l_{\mathrm{tw}}^{\mathrm{hel}}$ persistence lengths of a helix. Asymmetric neutralization of charges upon adsorption of the helix on the sphere would soften the helix, and consequently, bending and twisting become nonuniform along the helix. Below, however, we consider pure bending of the helix, with no charge neutralization on the helix upon its bending.

Recently, similar calculations have been performed in refs 27,41 , and 42 . For instance, in ref 41 it was shown that the electrostatic energy of the helix is lower than the one of a line with the same linear charge density (because the charges are better separated), and $l_{\mathrm{el}}^{\text {hel }}$ is typically larger than that of the line, $l_{\mathrm{el}}^{\text {line }}$. Within the same Debye-Hückel model the electrostatic cost of twist deformations of a DNA-like double spiral has been calculated in ref 42 . It was shown that the $l_{\mathrm{tw}}^{\text {hel }}$ can change sign, depending on the environmental conditions and structural parameters. We adapt a similar approach and our results are quite similar to those of refs 41 and 42 .

Electrostatic Energy of the Helix. We consider a single helix of discrete charges on the surface of a rod with the same dielectric constant and the screening length in the rod interior and exterior, similarly to refs 41 and 42 . The electrostatic energy of the helix $E_{\mathrm{el}}$ of radius $a$ and helical pitch $H,{ }^{48,49}$ with the radius of curvature $R_{\mathrm{c}}$, can then be approximated within the same Debye-Hückel limit as (per one charge)

$$
E_{\mathrm{el}}\left(R_{\mathrm{c}}, H, a, h\right) /\left(k_{\mathrm{B}} T l_{\mathrm{B}}\right)=\sum_{n=1}^{\infty} \frac{\mathrm{e}^{-\kappa \mathrm{r}_{n}}}{r_{n}}
$$

where the $r_{n}$ are now the distances between the charges on a smoothly bent spiral (spiral on the surface of a toroid). The bending contracts the charge separations on the inner radius, and it stretches the charge separations on the outer radius of the bent helix. That might cause a difference between the electrostatic persistence length of the helix and of the linear array of charges.

In the limit of thin helices, $a / h \lesssim 1$ (it can work quite well up to $a \sim h$ ), and for large curvature radius, the energy of a bent helix is (per one charge)

$$
\begin{aligned}
\frac{E_{\mathrm{el}}}{k_{\mathrm{B}} T} \approx & l_{\mathrm{B}} \sum_{n=1}^{\infty}\left\{\frac{\mathrm{e}^{-\kappa n h}}{n h}+\frac{\mathrm{e}^{-\kappa n h} n h(1+\kappa n h)}{24 R_{\mathrm{c}}^{2}}-\right. \\
& \left.a^{2} \mathrm{e}^{-\kappa n h} \frac{1+\kappa n h}{n^{3} h^{3}} 2 \sin ^{2}\left[\frac{n g h}{2}\right]\right\}+\frac{\Delta l_{\mathrm{el}}^{\mathrm{hel}}(a, H, h, \kappa)}{2 R_{\mathrm{c}}{ }^{2}}
\end{aligned}
$$

The intrinsic reciprocal screening length emerging from the helical charge distribution is $g=2 \pi / H$. The first term in this expression is the energy of a linear array of charges with the axial charge-charge separation $h$ (eq 1). The second term in (10) is the energy required to bend this array. Equation 4 for $l_{\mathrm{el}}^{\text {line }}$ follows from this term after the summation. The third term is a negative correction to the energy of a straight array of charges due to the finite radius of the helix. The fourth term is a correction to the bending energy of a linear array of charges accounting for the charge helicity; $\Delta l_{\mathrm{el}}^{\mathrm{hel}}$ is the correction to $l_{\mathrm{el}}^{\text {line }}$. Higher order terms, $O\left(a^{4}, R_{\mathrm{c}}^{-4}\right)$, have been omitted in eq 10.

Straight Helix. At $R_{\mathrm{c}} \rightarrow \infty$ we have $r_{n}=$ $\sqrt{n^{2} h^{2}+4 a^{2} \sin ^{2}[n g h / 2]}$, and the energy of the straight helix can be written as (per one charge)

$$
\begin{aligned}
& \frac{E_{\mathrm{el}}^{\mathrm{hel}}(H, a, h)}{k_{\mathrm{B}} T l_{\mathrm{B}}}=-\frac{\ln \left[1-\mathrm{e}^{-\kappa h}\right]}{h}- \\
& \frac{a^{2}}{2 h^{3}}\left\{2 P_{3}\left[\mathrm{e}^{-\kappa h}\right]-P_{3}\left[\mathrm{e}^{-\kappa h+i g h}\right]-P_{3}\left[\mathrm{e}^{-\kappa h-i g h}\right]\right\}- \\
& \frac{a^{2} \kappa}{2 h^{2}}\left\{2 P_{2}\left[\mathrm{e}^{-\kappa h}\right]-P_{2}\left[\mathrm{e}^{-\kappa h+i g h}\right]-P_{2}\left[\mathrm{e}^{-\kappa h-i g h}\right]\right\}
\end{aligned}
$$

with $P_{m}[Z]=\sum_{n=1}^{\infty} Z^{n} / n^{m}$ the polylogarithm function. Figure 5a shows that $E_{\mathrm{el}}^{\text {hel }}<E_{\mathrm{el}}^{\text {line }}$ because the separation of charges on the helix is more favorable and thus their repulsion is weaker. The corresponding energy difference is $\propto a^{2}$. Above some value of $a$, of course, this tendency should break down, together with the approximation of thin helices ( $E_{\mathrm{el}}^{\mathrm{hel}}$ must be positive). Note that $E_{\mathrm{el}}^{\text {hel }}$ diverges at $\kappa \rightarrow 0$ since the sum in (1) with the nonscreened Coulomb potential does not converge.

Discreteness of Charges. The dependence $E_{\mathrm{el}}^{\mathrm{hel}}(H)$ on the pitch $H$ at constant $h$ is presented in Figure $5 \mathrm{~b}$ (the number of charges per helical pitch is not constant). The exact electrostatic energy, obtained by numerical summation of eq 9 , has a minimum at $H \approx 2 h$. In this case, the separation between the nearest charges along the helix is maximal: the charges are located on the opposite sides of the helix (Figure 1c). The energy of the helix with $H=h$ and $H=\infty$ is equal to the energy of the line with the same $h$. Figure $5 \mathrm{~b}$ shows that the approximate 

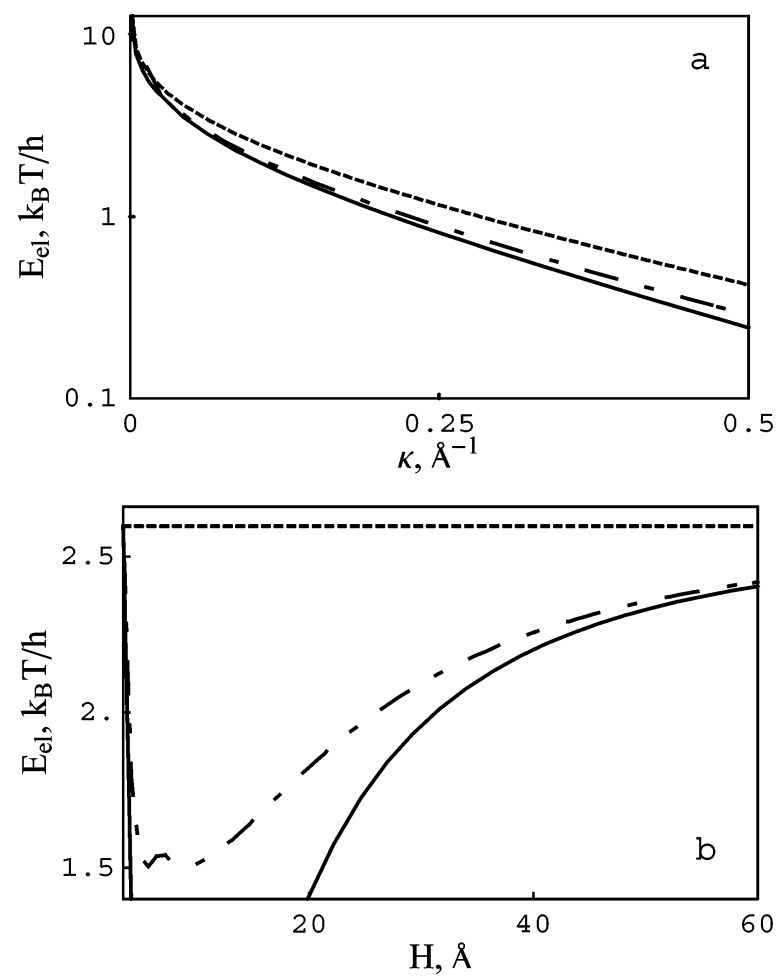

Figure 5. Electrostatic energy of the linear array of charges, $E_{\mathrm{el}}^{\text {line }}$ (dotted, eq 1), and the energy of the helix, $E_{\mathrm{el}}^{\mathrm{hel}}$, calculated via direct numerical summation (dotted-dashed, eq 9 ) and approximately (solid, eq 11). Parameters: $a=3 \AA, h=3.4 \AA, l_{\mathrm{B}}=7.1 \AA ; H=10 h$ (part a) and $\kappa=0.1 \AA^{-1}$ (part b).

energy expression, eq 11, works quite well for large $H$. For $H$ $\gtrsim 2 h$ the energy of the helix grows with $H$ that makes the overtwisting of the helix favorable from the point of view of electrostatics because the charge separation becomes larger (this energy growth follows also from eq 9 of ref 42). ${ }^{55}$

The strategy of calculating $E_{\mathrm{el}}^{\mathrm{hel}}$ via summation of DebyeHückel potentials has been suggested by Manning. ${ }^{27,41}$ In general, however, the final summation in eq 9 cannot be performed. In the limit of thin helices, this is possible, and it gives an energy value rather close to the exact result for $a \lesssim h$, as shown in eq 11 and Figure 5a. The figure provides results for a helix with the linear charge density of $B$-DNA but with $a$ $=3 \AA$, where the approximation of a thin helix applies. Note that for smaller $\kappa$ more terms have to be taken into account in the numerical evaluation of the sum in eq 10 in order to achieve the same accuracy because further apart charges interact more strongly.

Twisting. Using eq 11, we can estimate the electrostatic contribution to the helix twist elasticity. We impose a small uniform twist $\omega$ to the helix with pitch $H$ and expand the energy difference in powers of $\omega$

$$
\delta E / h=\left[E_{\mathrm{el}}^{\mathrm{hel}}(g+\omega)-E_{\mathrm{el}}^{\mathrm{hel}}(g)\right] / h \approx W_{\mathrm{tw}}^{\mathrm{hel}} \omega+k_{\mathrm{B}} T l_{\mathrm{tw}}^{\mathrm{hel}} \omega^{2} / 2
$$

The second derivative of $\delta E$ with respect to $\omega$ yields

$$
\begin{aligned}
& l_{\mathrm{tw}}^{\mathrm{hel}}(H, h, a)=\frac{\partial^{2}(\delta E / h)}{\partial \omega^{2}} \simeq \\
& \frac{a^{2} l_{\mathrm{B}}}{2 h^{2}}\left\{\ln \left[\frac{\cosh [\kappa h]-\cos [g h]}{\mathrm{e}^{\kappa h} / 2}\right]+\frac{\kappa h\left(\mathrm{e}^{-\kappa h}-\cos [g h]\right)}{\cosh [\kappa h]-\cos [g h]}\right\}
\end{aligned}
$$

The helicity, in the thin helix approximation, gives a small cor-
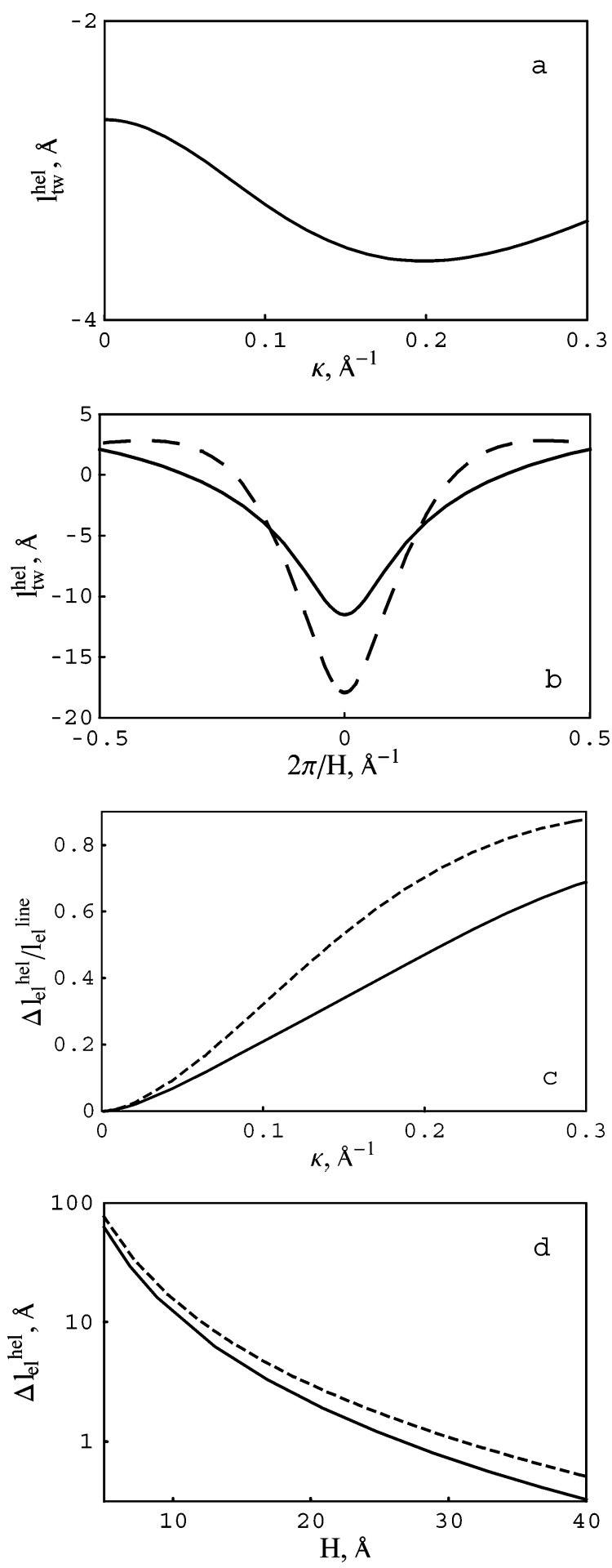

Figure 6. Electrostatic twisting (a, b), eq 13, and bending (c, d), eq 15 , persistence lengths of a helical array of charges. Parameters for part a (b) are the same as in Figure 5a (5b), respectively; for part c $a$ $=3 \AA, H=4 h=34 \AA$ and for part d $a=3 \AA, H=4 h, \kappa=0.1 / \AA$. In part $\mathrm{b}$ the solid curve is eq 13; the dashed curve is $l_{\mathrm{tw}}^{\text {hel }}$ obtained in ref 42. In parts $\mathrm{c}$ and $\mathrm{d}$ the solid curves are caculated according to eq 15; the dotted curves are the results of ref 41 for small $a$.

rection to the mechanical DNA twisting persistence length, $l_{\mathrm{tw}}$ $\sim 750 \AA^{51,52}$ (Figure 6a). Since $l_{\mathrm{tw}}^{\mathrm{hel}} \propto a^{2}$, it can reach a considerable fraction of $l_{\mathrm{tw}}$ for $a \sim 10 \AA$. The dependence $l_{\mathrm{tw}}^{\mathrm{hel}}(\kappa)$ is relatively weak (Figure 6a), as it was also predicted in ref 42 .

Figure $6 \mathrm{~b}$ shows that $l_{\mathrm{tw}}^{\text {hel }}$ assumes positive and negative values depending on the pitch. In the limit $H \rightarrow \infty$ at constant $h$, eq 13 yields $l_{\mathrm{tw}}^{\text {hel }}<0$ since the corresponding helix has the 
highest energy (Figure 5b), and thus it is unstable with respect to decreasing $H$. Similarly, for $H \approx 2 h$ where the energy assumes a minimum, the helix is stable and $l_{\mathrm{tw}}^{\text {hel }}>0$. Such a change of sign has recently been predicted within a similar model in ref 42 (dashed curve in Figure 6b).

For the parameters used in Figure 6b, the approximation of thin helices reaches beyond the region of its applicability when $|2 \pi / H| \gtrsim 0.3$. However, the shape of $l_{\mathrm{tw}}^{\mathrm{hel}}(H)$ is the same both for small $a$ (where the approximation works) and for large $a$ because $l_{\mathrm{tw}}^{\mathrm{hel}} \propto a^{2}$. Thus, negative values of $l_{\mathrm{tw}}^{\text {hel }}$ are unlikely to be an artifact of the chosen approximations. Note that although for higher salt concentrations $E_{\mathrm{el}}^{\mathrm{hel}}$ decreases rapidly, $l_{\mathrm{tw}}^{\text {hel }}$ changes only slightly with $\kappa$.

Bending. The electrostatic bending persistence length of the helical array of charges in a solution with simple salt

$$
l_{\mathrm{el}}^{\text {hel }}=l_{\mathrm{B}} /\left(4 \kappa^{2} h^{2}\right)+\Delta l_{\mathrm{el}}^{\mathrm{hel}}
$$

can also be calculated. We show below that the correction $\Delta l_{\mathrm{el}}^{\mathrm{hel}}$ can be both positive and negative.

Intuitively, there is a simple argument why the helix can be bent easier than the linear array of charges with the same axial charge-charge separation $h$. Let us take a helix with a large radius and small pitch such that $H \lesssim \kappa^{-1} \lesssim a$ and, for instance, $H=4 h$. (About $80 \%$ of the bare DNA charge is typically neutralized by the condensed cations; ${ }^{44}$ i.e., only about four charges per DNA pitch remain noncompensated.) Thus, the bent helix consists of four bent linear arrays, each with the intercharge separation $H$. For the parameters chosen, the repulsion is considerable between the charges within the same array, but it is negligible between the charges of different arrays. Here, one can write for the persistence length of the helix that $l_{\mathrm{el}}^{\text {hel }} \approx 4 l_{\mathrm{B}} /$ $\left(4 \kappa^{2} H^{2}\right)$, whereas for the initial linear array $l_{\mathrm{el}}^{\text {line }}=l_{\mathrm{B}} /\left(4 \kappa^{2} h^{2}\right)$; i.e., nearly a 4 times larger value is found. This limit is, however, opposite to the limit of thin helices considered in the present paper, when the helix radius is the smallest parameter of the problem.

The general expression for $l_{\mathrm{el}}^{\mathrm{hel}}$ has been obtained in ref 41 ; the summation in the final expression however cannot be performed for arbitrary helix parameters. Using the Taylor expansions of eqs 19 and 20 of ref 41 for small helical radius $a$, one obtains that a thin helix is harder to bend than the linear array of charges. Doing the same expansion for the helix with four charges per pitch one can get an estimation

$$
\begin{array}{r}
\Delta l_{\mathrm{el}}^{\mathrm{hel}} \simeq \frac{a^{2} l_{\mathrm{B}}}{6 h} \sum_{n=1}^{\infty} \frac{\mathrm{e}^{-\kappa n h}}{n h}\left\{\left(3+3 \kappa n h+\kappa^{2} n^{2} h^{2}\right) 2 \cos ^{2}[n g h / 2]+\right. \\
\left.n^{2} h^{2} \kappa^{2} \cos [n g h]\right\}
\end{array}
$$

At physiological salt concentrations and below, the corrections to $l_{\mathrm{el}}^{\text {line }}$ due to the charge helicity are small (Figure 6c). These corrections affect only slightly the results of the model of sphere overcharging by the wrapped rod, presented in section II. The dependence of $\Delta l_{\mathrm{el}}^{\mathrm{hel}}$ at small $a$ on the ionic strength of the solution and on the helical pitch is very similar for eq 15 and for expressions presented in ref 41 (Figure 6c,d).

Experiments show that the DNA bending modulus is strongly $\kappa$ dependent, ${ }^{53}$ whereas the DNA twist modulus is almost insensitive to $\kappa^{52}$ which is consistent with our predictions. Note that these results, obtained in the limit of large curvature radii, are not directly applicable to the DNA wrapping in the nucleosome, where the DNA curvature radius is as small as 5 $\mathrm{nm}$; the DNA radius also cannot be considered as small.
Note that the low-dielectric core of DNA might enhance the electrostatic interactions considerably; $l_{\mathrm{tw}}^{\mathrm{hel}}$ and $l_{\mathrm{el}}^{\mathrm{hel}}$ would then increase correspondingly. The precise value of $\epsilon$ inside the DNA and close to it, however, is unknown, and therefore we cannot make here any conclusive predictions for the DNA electrostatic twisting and bending rigidity. The water-DNA dielectric boundary should also modify the Debye-Hückel screening law between the charges used in the present paper. ${ }^{50}$

\section{Discussion and Conclusions}

As outlined in the Introduction, a considerable overcharging of the NCP by a DNA wrapped around the histone core is well established; however, the reasons for this are not fully understood. In the first part of this paper, we have explored one of the possible overcharging mechanisms connected with the asymmetric neutralization of DNA charges by an oppositely charged sphere. Such neutralization induces a rod bending toward the macroion, resulting in its overcharging. In the second part, we have considered a helical array of charges on a bent DNA-like polyelectrolyte rod and have calculated the energy of the helix and the electrostatic contributions to its bending and twisting elasticity modules, in the limit of thin helices. These corrections are typically small and would modify only slightly the prediction of the sphere overcharging by the rod, presented in section II.

These findings might be relevant to description the NCP. The NCP crystal structure shows ${ }^{8}$ that within the NCP the histone proteins form a number of (electrostatic) contacts, hydrogen bonds, and salt links with the DNA phosphates. In addition, positively charged arginine chains enter the DNA minor groove every time when it faces the octamer, interacting directly with the DNA phosphates. ${ }^{8,56}$ Possible charge neutralization caused by these interactions and the repulsion of noncompensated outerto-core DNA phosphates can contribute to NCP overcharging. Also, this can result in a nonuniform DNA "kinking" in the NCP which occurs in special positions with respect to the histones. $^{8}$

DNA-DNA Electrostatic Interaction in the Nucleosome. The interaction between the DNA turns, neglected in the present paper, likely also contributes to NCP stability. Below we discuss possible consequences of this interaction and analyze them in terms of known theories of DNA-DNA interactions.

In particular, it is known that electrostatic interactions between the nearest DNA turns should stabilize the NCP.5,6 It was suggested that in the NCP a "bridging of negatively charged phosphate groups on the DNA by means of cations or positively charged histone side chains" can occur. ${ }^{5}$ For a repetitive formation of such bridges along the whole length of the wrapped DNA, it was suggested that an integer number of bp per turn of wrapped DNA would be favored. ${ }^{5}$ It was also shown that each DNA turn around the histone core contains $\approx 7.6$ DNA helical pitches: as a result of this periodicity, "adjacent turns of the superhelix are arranged with a minor groove approximately opposite to the major groove". ${ }^{6}$ Later experimental studies have shown that highly charged $\mathrm{H} 2 \mathrm{~B}$ as well as $\mathrm{H} 3$ histone tails often pass through the aligned minor grooves of the nearest DNA turns, connecting the DNA turns together via the "bridges". 8 I.e., the tails "zip" oppositely charged DNA minor grooves together that, together with the common electrostatic DNA-histone attraction, is expected to influence the stability of the NCP.

Recent NCP crystallography studies ${ }^{8,18}$ have indicated that another zipping mechanisms might also be involved in NCP stabilization. It comes from groove-strand alignment of nearest 
turns of the DNA superhelix (Figure 1a in ref 8). Similarly, in columnar DNA assemblies, the attraction between $B$-DNA duplexes has been predicted to occur on the basis of the Kornyshev-Leikin theory of DNA-DNA electrostatic interactions. ${ }^{57,58}$ This attraction is due to the "electrostatic zipper" formed between the strands of one DNA and the adsorbed cations residing in the grooves of another DNA. ${ }^{57,58}$ In this theory, the DNA phosphates and the adsorbed cations are treated as spirals of charges on the surface of a rod with a low dielectric constant representing the DNA core. The adsorption of (multivalent) cations into the DNA grooves was shown to enhance this zipperlike attraction. ${ }^{58,59}$ For two parallel $B$-DNA duplexes at $27 \AA$ interaxial separation the possible gain of the electrostatic interaction energy can be as large as $\sim 15 k_{\mathrm{B}} T$ per $50 \mathrm{bp}$, at optimal azimuthal alignment of DNAs. ${ }^{58}$ Aligned fragments of a DNA superhelix in the NCP are expected to obey the interaction laws similar to those of parallel DNA helices. ${ }^{57,58,60}$ Thus, the energy of NCP stabilization coming from the electrostatic interaction of aligned DNA fragments in the NCP can be also $\sim 15 k_{\mathrm{B}} T(3 / 4$ of the superhelical turn, about $50 \mathrm{bp}$, is the length of DNA-DNA contacts in the NCP). This energy gain grows with the length of DNA-DNA contacts that favors the DNA adsorption on the histone core and can thus also contribute to NCP overcharging.

Nucleosome and Dense DNA Aggregates. For two parallel DNAs, the Kornyshev-Leikin theory predicts that DNA-DNA contacts are stronger if the DNAs have an integer number of base pairs per DNA helical turn. ${ }^{57,61}$ The energy gain for a pair of ideal $B$-DNA duplexes was shown to be nearly twice as large as the torsional energy of DNA overtwisting by $0.5 \mathrm{bp} / \mathrm{pitch}$ (the latter is $\sim 1 k_{\mathrm{B}} T$ per DNA pitch) at DNA-DNA separations relevant to NCP. This electrostatic preference is likely to be the reason for the DNA overwinding from $10.5 \mathrm{bp} / \mathrm{pitch}^{11}$ to $10.0 \mathrm{bp} / \mathrm{pitch}^{62,63}$ upon transition from dilute solutions to dense assemblies/fibers. ${ }^{61}$ One can speculate that, similarly, the DNA overtwisting to about $10.0 \mathrm{bp} /$ turn observed in the $\mathrm{NCP}^{8,10}$ is required for a better alignment of nearest DNA turns of the superhelix because "then the same stabilizing interactions between adjacent superhelix turns can occur repeatedly along the (DNA) chain". 5

Other similarities in the behavior of DNA wrapped on the NCP and of DNA dense aggregates indicate the importance of DNA-DNA electrostatics. ${ }^{64}$ The average DNA-DNA interaxial separation in the NCP is about $27 \AA,{ }^{1,6,8}$ which is close to the separation at which the DNA-DNA attraction in the presence of condensing cations is detected in DNA columnar assemblies ${ }^{65}$ and in DNA-cationic lipid complexes ${ }^{67}$ as well as to the equilibrium DNA-DNA separation in toroidal DNA condensates; ${ }^{66,68}$ theoretically, DNA-DNA attraction at such separations has also been predicted on the basis of the theory of electrostatic interaction of DNA duplexes. ${ }^{57-60}$ Some properties of condensation and resolubilization of NCPs in the presence of biologically important di- and trivalent ions ${ }^{5,69}$ are also similar to those observed for free DNA in solution, ${ }^{65,70}$ indicating the importance of DNA-DNA electrostatic interactions in both cases. $^{71}$

The higher-order structures of the NCPs also involve interactions between DNA fragments wrapped on different NCPs. Pulling a single chromatin fiber ${ }^{72}$ has revealed, for instance, that the internucleosomal interaction energy in the fiber is $\approx 3 k_{\mathrm{B}} T$ per nucleosome. (The internucleosomal contacts in the fiber appear to disrupt at the pulling force of about $5 \mathrm{pN}$.) The stability of the $30 \mathrm{~nm}$ nucleosome fiber is triggered by addition of lysinerich $\mathrm{H} 1$ proteins, ${ }^{73}$ known to interact with wrapped DNA when it leaves and enters the NCPs (polylysine is known to condense free DNA efficiently ${ }^{74}$ ). The interaction of the highly charged histone tails outside the core (not visible in the crystal structure) with the DNA can also contribute to the stability and influence the structure of the $30 \mathrm{~nm}$ fiber. ${ }^{75}$

Outlook. Although a theory of electrostatic interactions within the NCP is not constructed yet, the (oversimplified) electrostatic model suggested in the present paper may clarify some of the relevant points. Advanced studies might involve further peculiarities of DNA-DNA and DNA-histone interactions including the difference in their dielectric constants and possible specific/bridging interactions, sequence-dependent DNA deformability ${ }^{76,77}$ and periodicity, ${ }^{3}$ the influence of counterion condensation, ${ }^{44}$ and, probably, DNA-DNA chiral interactions. ${ }^{78}$ On a higher level of NCPs organization, the crystallization ${ }^{5,6,79}$ and folding of interconnected NCP filaments into high-order (solenoidal) structures $^{80-83}$ might also involve DNA-DNA interactions. For instance, the folding into the $30 \mathrm{~nm}$ solenoidal fiber can be triggered by addition of multivalent cations, which presumably adsorb into DNA grooves making the DNA-DNA electrostatic interactions more favorable. The influence of the DNA double-helical structure on DNA properties in macromolecular assemblies could be the subject of future investigations.

Acknowledgment. Helpful discussions with R. Golestanian, G. S. Manning, and F. Mohammad-Rafiee are acknowledged. After the major part of the present work has been finished, we have found ref 42 where the DNA electrostatic twisting elasticity modulus has been calculated. We thank F. Mohammad-Rafiee for providing some materials and details related to ref 42 , prior to publication.

\section{References and Notes}

(1) Saenger, W. Principles of Nucleic Acid Structure; SpringerVerlag: New York, 1984.

(2) Widom, J. Q. Rev. Biophys. 2001, 34, 269.

(3) Klug, A.; Lutter, L. C.; Rhodes, D. Cold Spring Harbor Symp. 1982, 47, 285.

(4) Kornberg, R. D.; Thomas, J. O. Science 1974, 184, 865. Kornberg, R. D. Science 1974, 184, 865.

(5) Finch, J. T.; Lutter, L. C.; Rhodes, D.; Brown, R. S.; Rushton, B.; Levitt, M.; Klug, A. Nature (London) 1977, 269, 29.

(6) Richmond, T. J.; Finch, J. T.; Rushton, B.; Rhodes, D.; Klug, A. Nature (London) 1984, 311, 532.

(7) Struck, M.-M.; Klug, A.; Richmond, T. J. J. Mol. Biol. 1992, 224, 253. Klug, A.; Rhodes, D.; Smith, J.; Finch, J. T.; Thomas, J. O. Nature (London) 1980, 287, 509.

(8) Luger, K.; Mäder, A. M.; Richmond, R. K.; Sangent, D. F.; Richmond, T. J. Nature (London) 1997, 389, 251.

(9) Rhodes, D. Nature (London) 1997, 289, 231.

(10) Klug, A.; Lutter, L. C. Nucleic Acid Res. 1981, 9, 4267.

(11) Rhodes, D.; Klug, A. Nature (London) 1980, 286, 573. Peck, L. J.; Wang, J. C. Nature (London) 1981, 292, 375. Rhodes, D.; Klug, A. Nature (London) 1981, 292, 378. Wang, J. C. Proc. Natl. Acad. Sci. U.S.A. 1979, 76, 200.

(12) Minsky, A. Annu. Rev. Biophys. Biomol. Struct. 2004, 33, 317 and references therein.

(13) Travers, A.; Klug, A. Nature (London) 1987, 327, 280.

(14) Crick, F. H. C.; Klug, A. Nature (London) 1975, 255, 530.

(15) Sobell, H. M.; et al. Proc. Natl. Acad. Sci. U.S.A. 1976, 73, 3068.

(16) Sussman, J. L.; Trifonov, E. N. Proc. Natl. Acad. Sci. U.S.A. 1978, $75,103$.

(17) In particular, the model of segmental kinking of nucleosomal DNA proposed in ref 14 suggests 10 bp DNA kink periodicity in NCP. As each kink imparts a substantial negative twist to DNA, in their model a kinked DNA adapts a left-handed superhelix,${ }^{14}$ being bent into the minor groove. The same kink periodicity was also revealed for the DNA with intercalated drugs, ${ }^{15}$ but with the bending into the major groove. Models of nonkinked DNA bending in NCP have also been considered. ${ }^{16}$

(18) Richmond, T. J.; Davey, C. A. Nature (London) 2003, 423, 145.

(19) Yager, T. D.; McMurray, C. T.; van Holde, K. E. Biochemistry 1989, 28, 2271. 
(20) Wang, H.; Bash, R.; Yodh, J. G.; Hager, G. L.; Lohr, D.; Lindsay, S. M. Biophys. J. 2002, 83, 3619.

(21) Mirzabekov, A. D.; Rich, A. Proc. Natl. Acad. Sci. U.S.A. 1979, 76,1118 .

(22) Manning, G. S.; et al. J. Biomol. Struct. Dyn. 1989, 6, 877.

(23) Kosikov, K. M.; Gorin, A. A.; Lu, X.-J.; Olson, W. K.; Manning, G. S. J. Am. Chem. Soc. 2002, 124, 4838.

(24) Manning, G. S. J. Am. Chem. Soc. 2003, 125, 15087.

(25) Schiessel, H. J. Phys.: Condens. Matter 2003, 15, R699 and references therein.

(26) Marky, N. M.; Manning, G. S. J. Mol. Biol. 1995, 254, 50; Biopolymers 1991, 31, 1543.

(27) Manning, G. S. Biophys. Chem. 2002, 101, 461.

(28) Schiessel, H.; Bruinsma, R. F.; Gelbart, W. M. J. Chem. Phys. 2001, 115,7245

(29) Netz, R. R.; Joanny, J.-F. Macromolecules 1999, 32, 9026

(30) Kunze, K.-K.; Netz, R. R. Phys. Rev. E 2002, 66, 011918.

(31) Nguyen, T. T.; Shklovskii, B. I. J. Chem. Phys. 2001, 115, 7298; 2001, 114, 5905. Grosberg, A. Yu.; Nguyen, T. T.; Shklovskii, B. I. Rev. Mod. Phys. 2002, 74, 329.

(32) Gurovich, E.; Sens, P. Phys. Rev. Lett. 1999, 82, 339. Golestanian, R. Phys. Rev. Lett. 1999, 83, 2473.

(33) Park, S. Y.; Bruinsma, R. F.; Gelbart, W. M.Europhys. Lett. 1999, $46,454$.

(34) Spakowitz, A. J.; Wang, Z.-G. Phys. Rev. Lett. 2003, 91, 166102.

(35) Cherstvy, A. G.; Winkler, R. G. Strong and weak adsorption of polyelectrolyte chain on an oppositely charged sphere, in preparation.

(36) Chodanowski, P.; Stoll, S. J. Chem. Phys. 2001, 115, 4951. Wallin, T.; Linse, P. J. Chem. Phys. 1998, 109, 5089. Wallin, T.; Linse, P. Langmuir 1996, 12, 305. Skepo, M. J. Phys. Chem. B 2004, 108, 5431.

(37) Akinchina, A.; Linse, P. Macromolecules 2002, 35, 5183. Akinchina, A.; Linse, P. J. Phys. Chem. B 2003, 107, 8011 and references therein.

(38) Winkler, R. G. New J. Phys. 2004, 6, 11.

(39) Winkler, R. G.; Steinhauser, M. O.; Reineker, P. Phys. Rev. E 2002, 68, 021802

(40) Kayitmazer, A. B.; et al. J. Phys. Chem. B 2003, 107, 8158.

(41) Manning, G. S. Macromolecules 2001, 34, 4650.

(42) Mohammad-Rafiee, F.; Golestanian, R. Phys. Rev. E 2004, 69, 061919.

(43) Manning, G. S. J. Phys. Chem. B 2003, 107, 11485.

(44) Manning, G. S. Q. Rev. Biophys. 1978, 11, 179.

(45) A more general expression for the electrostatic bending persistence length of the rod can be derived for arbitrary $\kappa h$ value; see ref 43 . The expression used below for the parameters chosen $(h=3.4 \AA)$ overestimates the exact result by less than $10 \%$ for $\kappa \lesssim 0.5 \AA^{-1}$. Thus, further we use a simpler formula, eq 4

(46) Odijk, T. J. Polym. Sci. 1977, 15, 477. Skolnick, J.; Fixman, M. Macromolecules 1977, 10, 944.

(47) Zandi, R.; Rudnick, J.; Golestanian, R. Phys. Rev. E 2003, 67, 061805 .

(48) Note that the energy of the continuous helical spiral has been calculated in ref 49 as an exact solution of the Poisson-Boltzmann equation in cylindrical geometry. This expression did not contain the charge discreteness and the bending of the helix. Below, thus, we use the approximate summation of the Debye-Hückel interaction potentials between all pointlike charges on the helix.

(49) Cherstvy, A. G.; Winkler, R. G. J. Chem. Phys. 2004, 120, 9394

(50) Netz, R. R. Phys. Rev. E 1999, 60, 3174.

(51) Vologodskii, A. V.; et al. J. Mol. Biol. 1992, 272, 1224. FrankKamenetskii, M. D.; et al. J. Biomol. Struct. Dyn. 1985, 2, 1005. Store, D.; Baldwin, R. J. Mol. Biol. 1983, 170, 957. Crothers, D. M.; Drak, J.; Kahn, J. D.; Levene, S. D. DNA bending, flexibility and helical repeat by cyclization kinetics. In Methods in Enzymology; Lilley, D. M. J., Dahlberg, J. E., Eds.; Academic Press: San Diego, 1992; Vol. 212B, p 3.

(52) Taylor, W. H.; Hagerman, P. J. J. Mol. Biol. 1990, 212, 363.

(53) Baumann, C. G.; Smith, S.; Bloomfield, V. A.; Bustamante, C. Proc. Natl. Acad. Sci. U.S.A. 1997, 94, 6185. Wenner, J. R.; et al. Biophys. J. 2002, 82, 3160.

(54) Taylor, W. H.; Hagerman, P. J. J. Mol. Biol. 1990, 212, 363.

(55) Note that both $E_{\mathrm{el}}^{\text {hel }}$ for a continuous spiral obtained as an exact solution of the linear Poisson-Boltzmann equation in ref 49 and $E_{\mathrm{el}}^{\text {hel }}$ for the helical array of discrete charges calculated in ref 42 have a minimum at $H=0$. This minimum is understandable for a continuous spiral: when $H$ decreases, the effective reciprocal screening length $\kappa_{n}=$ $\sqrt{\kappa^{2}+n^{2}(2 \pi / H)^{2}}$ grows, and the electrostatic interactions are weakened. ${ }^{49}$ Thus, the $l_{\mathrm{tw}}^{\text {hel }}$ found in ref 42 is always positive at small $H$, while the $l_{\mathrm{tw}}^{\text {hel }}$ calculated from eq 13 becomes negative $\left(E_{\mathrm{el}}\right.$ increases when $H$ decreases at $H \lesssim 2 h$, Figure $5 \mathrm{~b}$ ). This region is not shown in Figure $6 \mathrm{~b}$.

(56) Garsia-Perez, M.; Pinto, M.; Subirana, J. A. Biopolymers 2003, 69,432 .

(57) Kornyshev, A. A.; Leikin, S. J. Chem. Phys. 1997, 107, 3656. Erratum: J. Chem. Phys. 1998, 108 7035(E); Phys. Rev. Lett. 2001, 86, 3666.

(58) Kornyshev, A. A.; Leikin, S. Phys. Rev. Lett. 1999, 82, 4138.

(59) Cherstvy, A. G.; Kornyshev, A. A.; Leikin, S. J. Phys. Chem. B 2002, 106, 13362

(60) Cherstvy, A. G.; Kornyshev, A. A.; Leikin, S. J. Phys. Chem. B 2004, 108, 6508 .

(61) Kornyshev, A. A.; Leikin, S. Biophys. J. 1998, 75, 2513; Proc. Natl. Acad. Sci. U.S.A. 1998, 95, 13579.

(62) Zimmerman, S. B.; Pheiffer, B. H. Proc. Natl. Acad. Sci. U.S.A. 1979, 76, 2703.

(63) Langridge, R.; Wilson, H. R.; Hooper, C. W.; Wilkins, M. H. F.; Hamilton, L. D. J. Mol. Biol. 1960, 2, 19.

(64) Gelbart, W. M.; Bruinsma, R. F.; Pincus, P. A.; Parsegian, V. A. Phys. Today 2000, 53, 38.

(65) Leikin, S.; Rau, D. C.; Parsegian, V. A. Phys. Rev. A 1991, 44, 5272. Rau, D. C.; Parsegian, V. A. Biophys. J. 1992, 61, 260.

(66) Hud, N. V.; Downing, K. H. Proc. Natl. Acad. Sci. U.S.A. 2001, $98,14925$.

(67) Koltover, I.; Wagner, K.; Safinya, C. R. Proc. Natl. Acad. Sci. U.S.A. 2000, 97, 14046.

(68) Schellman, J. A.; Parthasarathy, N. J. Mol. Biol. 1984, 175, 313.

(69) (a) Leforestier, A.; Fudaley, S.; Livolant, F. J. Mol. Biol. 1999, 290, 481. (b) Mandenot, S.; Raspaund, E.; Tribet, C.; Belloni, L.; Livolant, F. Eur. Phys. J. E 2002, 7, 221. (c) Leforestier, A.; Dubochet, J.; Livolant, F. Biophys. J. 2001, 81, 2414.

(70) Bloomfield, V. A. Biopolymers 1991, 31, 1471. Bloomfield, V. A Curr. Opin. Struct. Biol. 1996, 6, 334 and references therein.

(71) In particular, the existence of multiple supramolecular organizations of NCPs in the presence of spermidine (columnar isotropic, columnar nematic, and columnar hexagonal structures) has been reported;69a the addition of salt was shown to destroy first the hexagonal order, then the nematic order, and then the columnar structure of NCPs assemblies. At several $\mathrm{mM}$ of spermidine (charge +3 ) the solution of isolated NCPs form columnar hexagonal phases. ${ }^{69}$ a The separation between the nearest NCPs on different columns varies from about 10 to $12 \mathrm{~nm}$; the corresponding surface-to-surface separations is $4-13 \AA$. (The interparticle separation in the NCP crystals is even smaller, $\sim 110 \AA{ }^{5}$ i.e., NCPs are very closely packed.) At such separations the DNA-DNA electrostatic attraction in solution of multivalent cations is known to occur. The NCPs are thus complexed into dense aggregates due to spermidine-mediated interactions between the DNA fragments wrapped around NCPs in different columns ("bridging") ${ }^{69 a}$ These interactions may also occur in the fiber of interconnected NCPs that can influence the compactification mechanism of the NCPs (solenoid vs zigzag). The solenoid, with pitch of about $110 \AA$, is likely to have more contacts between DNA on the NCPs situated on adjacent solenoidal turns than a zigzag structure (the precise structure of the fiber is not yet resolved). Note that without multivalent cations solutions of isolated NCPs form columnar lamellar mesophases where the histone tails are likely to produce the DNA-DNA attractions, connecting the NCPs in neighboring columns. ${ }^{69 \mathrm{c}}$ 127

(72) Cui, Y.; Bustamante, C. Proc. Natl. Acad. Sci. U.S.A. 2000, 97,

(73) Hansen, J. C. Annu. Rev. Biophys. Biomol. Struct. 2002, 31, 361

(74) Laemmli, U. K. Proc. Natl. Acad. Sci. U.S.A. 1975, 72, 4288.

(75) Schiessel, H. Europhys. Lett. 2002, 58, 140. Schiessel, H.; Gelbart, W. M.; Bruinsma, R. Biophys. J. 2001, 80, 1940. Mergel, B.; Everaers, R.; Schiessel, H. Phys. Rev. E 2004, 70, 011915.

(76) Nelson, H. C. M.; Finch, J. T.; Luisi, B. F.; Klug, A. Nature (London) 1987, 330, 221.

(77) Olson, W. K.; Gorin, A. A.; Lu, X.-J.; Hock, L. M.; Zhurkin, V. B. Proc. Natl. Acad. Sci. U.S.A. 1998, 95, 11163.

(78) Kornyshev, A. A.; Leikin, S. Phys. Rev. Lett. 2000, 84, 2537; Phys. Rev. E 2000, 62, 2576.

(79) Germond, J. E.; et al. Proc. Natl. Acad. Sci. U.S.A. 1975, 72, 1843

(80) Finch, J. T.; Klug, A. Proc. Natl. Acad. Sci. U.S.A. 1976, 73, 1897.

(81) Klug, A. From macromolecules to biological assemblies" (Nobel Lecture); Angew. Chem., Int. Ed. Engl. 1983, 22, 565.

(82) Woodcock, C. L.; Grigoryev, S. A.; Horowitz, R. A.; Whitaker, N. Proc. Natl. Acad. Sci. U.S.A. 1993, 90, 9021.

(83) Wedemann, G.; Langowski, J. Biophys. J. 2002, 82, 2847. 